\title{
Homing of chloromethylbenzoyl ammonia-labeled bone marrow mesenchymal stem cells in an immune-mediated bone marrow failure mouse model in vivo
}

\author{
Y. Xiao, Y. Wang, L. Li, Y.H. Li, Y. Pang, J.Y. Song and Z.J. Jiang \\ Hematology Department, \\ Guangzhou General Hospital of Guangzhou Military Command, \\ Guangzhou, Guangdong Province, China \\ Corresponding author: Y. Xiao \\ E-mail: jdxiao111@yeah.net
}

Genet. Mol. Res. 13 (1): 11-21 (2014)

Received June 25, 2013

Accepted October 5, 2013

Published January 3, 2014

DOI http://dx.doi.org/10.4238/2014.January.3.2

\begin{abstract}
Aplastic anemia is an abnormal immune reaction disease in which $\mathrm{T}$ lymphocytes destroy hematopoietic stem and progenitor cells because of immune hyperactivity. Bone marrow mesenchymal stem cells (BMSCs) have hematopoietic supporting and immune regulation functions. This study investigated BMSCs homing in mice transplantation models after bone marrow failure. BALB/c mice were randomly divided into three groups: normal control, bone marrow failure model, and BMSC transplantation group. Chloromethyl benzamidolabeled BMSCs of BALB/c mice were transplanted through tail vein injection in mouse models with bone marrow failure. Flow cytometry and histological fluorescence microscopy were used to observe the dynamic distribution of labeled cells in different tissues. Average survival time, peripheral blood, and bone marrow morphological features were observed in mice from each group. Twenty-four hours after tail vein infusion of BMSCs, positively labeled cells were observed
\end{abstract}


in the bone marrows of recipient mice, and the number of positive cells increased significantly at $72 \mathrm{~h}(\mathrm{P}<0.05)$. In dead or dying mice, white blood cells, hemoglobin, platelets, and bone marrow mononuclear cells were all significantly higher in the BMSC transplantation group than in the BMSCs of the model group $(\mathrm{P}<0.01)$. Mean survival time was significantly shorter in the bone marrow failure model group than in the transplantation group $(\mathrm{P}<0.05)$. These results confirmed that the major of BMSCs injected via tail vein could migrate to injured bone marrow tissues within 24-72 $\mathrm{h}$ in a mouse model of bone marrow failure. Furthermore, BMSCs can promote hematopoietic recovery, reduce the degree of bone marrow failure, and significantly prolong survival time.

Key words: Bone marrow failure; Chloromethyl benzamidobut; Mesenchymal bone marrow stem cells; Aplastic anemia

\section{INTRODUCTION}

Aplastic anemia (AA) is characterized by peripheral blood pancytopenia and bone marrow hematopoietic failure. Its pathogenesis involves immune dysfunction, an abnormal hematopoietic microenvironment, and destruction of hematopoietic stem and progenitor cells. Recent studies have revealed that bone marrow-derived mesenchymal stem cells (BMSCs) not only express a variety of hematopoietic cytokines, but also have hematopoietic supporting and immunomodulatory functions. The existing literature suggests that BMSCs have the potential to treat AA. Although some cases of clinical BMSC transplantations were shown to alleviate symptoms of AA patients (Fang et al., 2009; Gao et al., 2010; Xiao et al., 2013), the specific mechanisms of the effects of transplanted BMSCs remain unclear.

Homing migration and differentiation phenomena after BMSC transplantation are the keys to fully understanding these mechanisms. However, no studies on migration and homing of BMSCs in AA patients' bodies have yet been conducted. Therefore, the present study established protocols using a mouse model of immune-mediated bone marrow failure. The operation is simple, non-toxic to cells, and stable. Chloromethyl-benzamidodialkylcarbocyanine (CM-Dil), a tracer with a long enduring time, was used to label BMSCs. The distribution and homing dynamics of labeled BMSCs in the bone marrow failure mouse model were observed in vivo in order to evaluate therapeutic effects of BMSCs for AA.

\section{MATERIAL AND METHODS}

\section{Materials}

A randomized controlled animal experiment was performed using eight 10-week-old healthy specific pathogen-free (SPF)-grade female DBA/2 mice with an average body weight of $21.5 \pm 0.5 \mathrm{~g}$. The mice were purchased from the Shanghai Silaike Animal Experimental Center [certificate SCXK (Shanghai) 2007-0005]. These mice served as cell donors of the AA model. Eight-week-old SPF-grade inbred BALB/c male mice with an average body mass of $15.0 \pm 0.5 \mathrm{~g}$ were used for BMSC culture. Forty-five 8-9 week-old (half male, half female) SPF-grade inbred $\mathrm{BALB} / \mathrm{c}$ mice with an average body mass of $20.0 \pm 0.3 \mathrm{~g}$ were purchased from the Animal Experi- 
mental Center of Guangdong Province [certificate SCXK (Guangdong) 2008-0002]. Disposal of animals during experimental procedures conformed to standard guidelines for laboratory animals published by the Ministry of Science and Technology of the People's Republic of China in 2006.

\section{Grouping and intervention}

Forty-five BALB/c mice were individually identified and divided into three randomly controlled groups. There was no intervention in the blank control group $(\mathrm{N}=15)$. The model group $(\mathrm{N}=15)$ was injected with phosphate-buffered saline (PBS) after completion of bone marrow failure modeling. The transplantation group $(\mathrm{N}=15)$ was infused with CM-Dil-labeled BMSCs after modeling. All mice were housed in the same SPF-grade animal room.

\section{Modeling of mice with bone marrow failure}

The model was established according to previously published methods (Yao and $\mathrm{Li}$ 1991; Tang et al., 2010; Ma et al., 2011). DBA/2 mice were killed by the cervical dislocation method and were soaked in 7\% ethanol for 5 min. The neck, armpit, groin, popliteal, and mesenteric lymph nodes were removed using the aseptic method. RPMI-1640 culture medium was added to the tissues, which were repeatedly sheared into a paste, gently grinded, and filtered through a $200-\mu \mathrm{m}$ nylon mesh. Next, the paste was made into a single-cell suspension with a concentration of $1 \times 10^{10}$ cells/L. Trypan blue staining showed that lymphocytes comprised more than $95 \%$ of the living cells in the suspension. BALB/c mice were treated with gammaray irradiation $(5.0 \mathrm{~Gy})$ all over the body, and $0.5 \mathrm{~mL} \mathrm{DBA} / 2$ mouse lymphocyte suspension, containing $5 \times 10^{6}$ lymphocytes, was injected in the mouse tail vein within $4 \mathrm{~h}$ of the radiation procedure. Mice were placed in the SPF-grade animal room after the modeling procedure in order to monitor survival time after lymphocyte injection.

\section{Model success criteria}

Models were determined to be established successfully according to the following criteria: 1) pancytopenia, 2) bone marrow biopsy showed dysplasia or hypoplasia in multiple bone marrow sites, and 3) increase in the proportion of non-hematopoietic cells.

\section{BMSC isolation and culture}

Eight male BALB/c mice were anesthetized and killed and placed in $75 \%$ ethanol for $5 \mathrm{~min}$. The femur and tibia were removed under sterile conditions, and both ends were cut to expose the marrow cavity. Dulbecco's modified Eagle medium/Ham's F12 (DMEM/F12) medium was used to wash out the bone marrow with a $5-\mathrm{mL}$ syringe. The cell suspension was prepared, cells were counted, and then resuspended in 10\% fetal bovine serum and DMEM/F12 completed medium. The cells were inoculated in $25-\mathrm{cm}^{2}$ plastic flasks at a density of $2 \times 10^{6}$ cells $/ \mathrm{cm}^{2}$ and then placed in a $37^{\circ} \mathrm{C}$ humidified incubator with $5 \% \mathrm{CO}_{2}$ for culture. The medium was changed after $48 \mathrm{~h}$, and then every 3 days thereafter. Passaging was performed when the confluence of cells reached $90 \%$, at which point the medium was aspirated, and the cells were washed twice with PBS and trypsinized for $5 \mathrm{~min}$. Cells were then inoculated in $25-\mathrm{cm}^{2}$ plastic 
flasks (1:2) for culture. Third generation cells were used in subsequent BMSC experiments.

\section{Identification of BMSCs}

Four samples of $2 \times 10^{5} 3$ rd generation BMSCs were prepared with PBS. Fluorescently-labeled monoclonal antibodies of CD34, CD45, CD29, and CD44 were added to the cell samples. Flow cytometry detection was performed to evaluate antigen expression on the cell surface, and results were analyzed using the CellQuest software.

\section{CM-Dil-labeled BMSCs}

BMSCs were labeled according to manufacturer instructions, with slight modifications. Fifty microliters dimethyl sulfoxide was dissolved in $50 \mu \mathrm{g}$ CM-Dil crystalline particles, and poured into $1 \mathrm{~g} / \mathrm{L}$ storage solution. Third generation BMSCs $\left(1 \times 10^{6}\right)$ were resuspended in $1 \mathrm{~mL}$ of D-Hank's solution, to which $5 \mathrm{ng} / \mathrm{L} \mathrm{CM}$-Dil stock solution was added (Ma et al., 2011). Cells were incubated at $37^{\circ} \mathrm{C}$ for $5 \mathrm{~min}$ and at $4^{\circ} \mathrm{C}$ for $15 \mathrm{~min}$ in the dark. Next, cells were washed twice with PBS and centrifuged to remove any unbound CM-Dil dye (Kruyt et al., 2003). Labeled cells ( $5 \times 10^{5}$ ) were resuspended in $200 \mu \mathrm{L}$ of PBS. Meanwhile, similarly treated but unlabeled CM-Dil BMSC suspensions were set up as the negative control, and flow cytometry was used to detect the marked rate. The remainder of CM-Dil-labeled BMSCs was cultured for $24 \mathrm{~h}$, and five different horizons (x100) were randomly selected to calculate the labeling efficiency using an inverted fluorescence microscope. Cells were cultured for 14 days after observations, and an inverted fluorescence microscope was used to observe fluorescence intensity and labeling efficiency.

\section{BMSC proliferation ability}

Well-growing 3rd generation CM-Dil-labeled BMSCs and the single suspension of unlabeled BMSCs of the same generation (CM-Dil-labeled and unlabeled group, respectively) were evaluated for their proliferative capacity using the 3-(4,5-dimethylthiazol-2-yl)-5diphenyltetrazolium bromide (MTT) colorimetric method. The growth curve was later constructed.

\section{Infusion of BMSCs}

At the 6th day after modeling, $2 \times 10^{9}$ cells/L CM-Dil-labeled BMSCs were stored at $4^{\circ} \mathrm{C}$ in PBS in the dark. Cells $\left(1 \times 10^{6}\right.$ per mouse) were injected via the tail veins of transplantation mice group using the aseptic method with $0.5 \mathrm{~mL}$ of PBS. The model group received injections of only $0.5 \mathrm{~mL}$ of PBS.

\section{Flow cytometry detection of BMSCs homing to the bone marrow}

At $24 \mathrm{~h}$ and $72 \mathrm{~h}$ after infusion of cells, mice in each group ( $\mathrm{N}=3$ at each time point) were killed by cervical dislocation, femurs were removed under sterile procedures, and both ends were cut to expose the marrow cavity in order to wash out the bone marrow. Red blood 
cells in the cell suspension were removed using the hypotonic lysis method. PBS was added to adjust the concentration of nucleated cells to $2 \times 10^{9}$ cells/L. Two-hundred microliters of bone marrow mononuclear cell suspension was used to detect the distribution ratio with flow cytometry. The least analysis mode of $10^{6}$ events per time period was used to detect the proportion of CM-Dil-labeled cells in the bone marrow. Data were processed with Cell Quest analysis software.

\section{Fluorescence microscopy of in vitro distribution of BMSCs}

Bone marrow cells were smeared, and lung, liver, and spleen tissues were immediately frozen and sliced into $8-\mu \mathrm{m}$-thick sections. Overall, 10 slices were obtained, which were fixed in ice acetone for $20 \mathrm{~min}$, washed 3 times with PBS for $3 \mathrm{~min}$, dyed with 1:200 DAPI for $1 \mathrm{~min}$ in the dark at room temperature, and then washed another 3 times with PBS for 3 min. The anti-quenching sealing medium was used to fix cells. Fluorescence intensity and the distribution of BMSCs in various tissues were observed, photographed, and recorded under the fluorescence microscope.

\section{Blood cell counts and peripheral blood smears}

The model group was in a moribund state. At the 14th day after modeling of the transplantation group, $20 \mu \mathrm{L}$ blood was taken from the tail vein for the cell count, and $10 \mu \mathrm{L}$ blood was collected for the peripheral blood smear, staining, and oil microscope observation.

\section{Bone marrow mononuclear cell count and bone marrow biopsy}

At the 14th day after modeling, remaining mice in each group were killed by cervical dislocation after blood extraction. Bone marrow cells were removed from the right femur with RPMI 1640 culture liquid. The hypotonic lysis method was used to remove red blood cells and the remaining cells were counted. The left femurs of mice were fixed, decalcified, paraffinembedded, semi-thin sliced, and hematoxylin-eosin stained. The degree of myeloproliferation, the hematopoietic tissue capacity, the fat cell content, and the sinusoid structure of the bone marrow, were all observed under the microscope.

\section{Main outcome measurements}

The general condition, changes in peripheral blood and the bone marrow, and the BMSC distribution in the body were observed and recorded for each group of mice.

\section{Statistical analysis}

Continuous variables are reported as means $\pm \mathrm{SD}$. All statistical analyses were performed by the same individual (Y. Xiao) using the SPSS 13.0 software. Differences between groups were assessed with the Student $t$-test of independent samples, and one-way analysis of variance was used for comparisons of multiple means. Kaplan-Meier generating curves were constructed, which were compared using the log rank test. Differences at $\mathrm{P}<0.05$ were considered to be statistically 
significant. Data from all 45 BALB/c mice were included in the final analysis of the experiment.

\section{RESULTS}

\section{BMSC morphology}

At $48 \mathrm{~h}$ after incubation, observations under the inverted phase contrast microscope revealed that adherent BMSCs were single with round morphologies. After 4-5 days, the typical BMSC clone hyperplasia shape was formed, and as the number of cells gradually increased, the morphology became more fusiform, triangular, or star-shaped. By the 7th day of primary cell culture, cells rapidly proliferated, and the swirling colony was formed. On the 10th day, each small colony formed large colonies containing hundreds of cells, with clear overlap and fusion at the junction of colonies. The cells were purified after passage, and spindle cells comprised more than $98 \%$ of total cells.

\section{BMSC immune phenotyping}

Flow cytometry was used to detect phenotypes of 3rd generation BMSCs. Results showed high expression level of the BMSC-specific antigens (95.45\% CD29 and 99.99\% CD44) and low hematopoietic antigen expression (1.06\% CD34 and 1.20\% CD45).

\section{CM-Dil-labeled BMSC observation in vitro}

Twenty-four hours after labeling BMSCs with CM-Dil, 95\% of the cells showed significant red fluorescence with the green light excitations under the inverted fluorescence microscope (Figure 1).

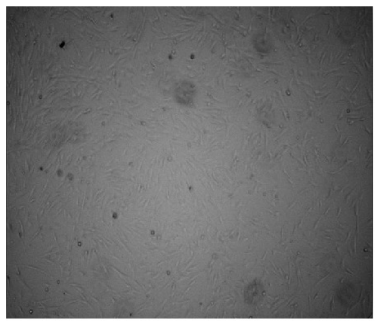

A

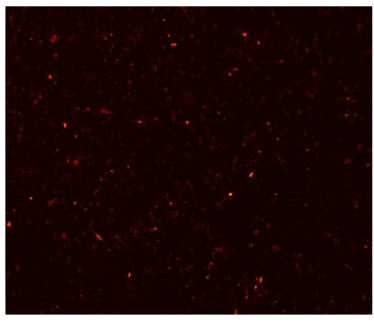

B

Figure 1. Bone marrow mesenchymal stem cells at $24 \mathrm{~h}$ after chloromethy-benzamidodialkylcarbocyanine labeling under inverted fluorescence microscope (100X). A. Under the light field of vision; B. under the dark field vision.

Adherent cells were mainly observed in fusiform and fibroblast-like shapes. In order to maintain good shape, early cell morphology displayed annular fluorescence with many fluorescent particles and high fluorescence intensity in the cells, but the nucleus was not fluorescently stained. After the subculture, the fluorescence faded slightly, but the red fluorescence remained clearly visible after 14 days of the marking. Flow determination of CM-Dil-labeled BMSCs revealed that the fluorescent labeling rate was $95.55 \%$ (Figure 2). 


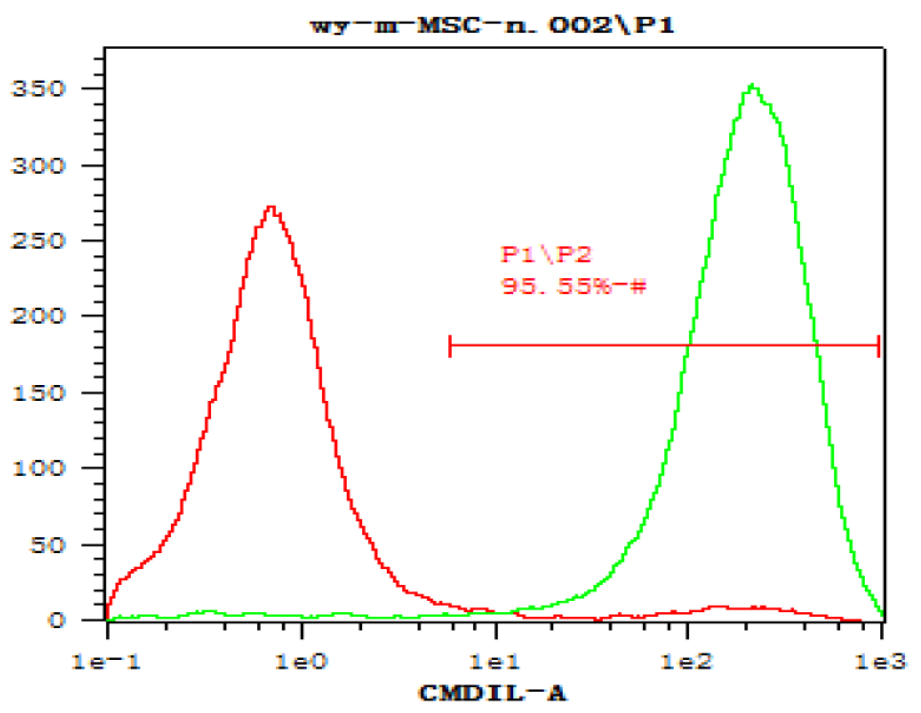

Figure 2. Flow cytometric analysis of the positive chloromethyl-benzamidodialkylcarbocyanine-labeled cell proportion in bone marrow mesenchymal stem cells.

\section{Cell proliferation changes in the BMSC labeled and unlabeled groups}

The growth curves of the two cell groups (labeled and unlabeled) were essentially similar. The first and second days were characterized by relatively slow growth, which corresponds to the latent growth period. The period from 3-7 days was characterized by a faster growth rate, corresponding to the logarithmic growth phase, and cells entered the growth platform period thereafter. At each time period after inoculation, proliferation of the CM-Dil-labeled cells was slightly lower than that of the unlabeled group, but the difference was not statistically significant $(\mathrm{P}=0.95)$.

\section{General changes in mice}

As of the second day, model mice showed symptoms of malaise, anorexia, body weight loss, significant activity decrease, arched shape, and piloerection. All model mice (N =9) were dead within the first 2 weeks. Anatomical investigation revealed that various organs were pale, thymus, spleen, and lymph node atrophy, and small intestine bleeding points were observed in some mice. Four of the 9 mice died after transplantation, and the survivors stabilized and showed increased activity up to the 10th day. The average survival time of the model group ( $11.00 \pm 0.33$ days) was lower than that of the transplantation group (13.22 \pm 0.31 days), and comparison of Kaplan-Meier survival function curves showed that the difference was statistically significant $(\log$ rank statistic $=11.726 ; \mathrm{P}=0.001)$.

\section{Peripheral blood changes in mice}

As shown in Figure 3, the peripheral blood smear in the blank control group showed hyperplasia and a normal number of nucleated cells. The peripheral blood smear in the model- 
ing group showed a significant reduction in the number of nucleated cells. Compared with the model group, the transplant group showed relatively higher hyperplasia activity and visible nucleated cells. Peripheral blood white blood cells, hemoglobin, and platelets were all significantly reduced in dying mice of the model group compared with the transplant and blank control groups $(\mathrm{P}<0.01)$ (Table 1).

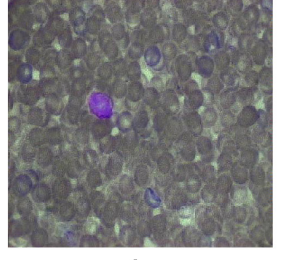

A

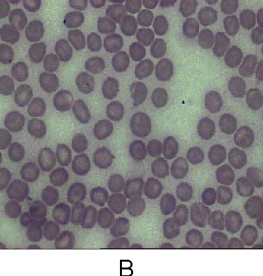

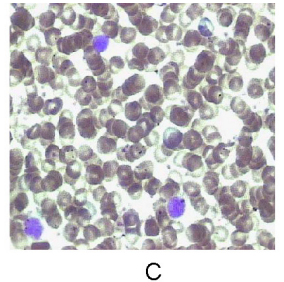

$\mathrm{C}$

Figure 3. Peripheral blood smears. A. Normal control mice; B. bone marrow failure mice; C. transplantation mice.

Table 1. Comparison of blood routine indices of dying or executed mice between groups $( \pm s, N=9)$.

\begin{tabular}{lccc}
\hline Group & White blood cells $\left(10^{9} / \mathrm{L}\right)$ & Hemoglobin $(\mathrm{g} / \mathrm{L})$ & Platelets $\left(10^{9} / \mathrm{L}\right)$ \\
\hline Normal control & $8.40 \pm 0.88^{\mathrm{a}}$ & $153 \pm 8^{\mathrm{a}}$ & $897 \pm 33^{\mathrm{a}}$ \\
Bone marrow failure & $0.21 \pm 0.18^{\mathrm{b}}$ & $63 \pm 7^{\mathrm{b}}$ & $15 \pm 6^{\mathrm{b}}$ \\
BMSC transplantation & $1.77 \pm 0.57$ & $94 \pm 12$ & $98 \pm 18$ \\
\hline
\end{tabular}

${ }^{\mathrm{a} C o m p a r i s o n}$ between bone marrow failure group and BMSCs transplantation group $(\mathrm{P}<0.01) .{ }^{\mathrm{b}}$ Comparison between normal control group and BMSCs transplantation group $(\mathrm{P}<0.01)$.

\section{Effects of BMSCs on bone marrow function}

Before dying or being killed, the number of mononuclear cells in unilateral bone marrows of transplanted mice $\left(0.17 \times 10^{7} \pm 0.01 \times 10^{7}\right)$ was significantly higher $(\mathrm{P}<0.01)$ than that of the model group $\left(0.03 \times 10^{7} \pm 0.02 \times 10^{7}\right)$. Several histopathological changes were observed in the bone marrow. The structure of the hematopoietic tissue in the blank control group was integrated, the hematopoietic cells were evenly distributed, and the hematopoietic cell proliferation was good. In dying model mice, myeloproliferation was extremely low, the number of nucleated cells was significantly reduced, and the number of non-hematopoietic cells increased. Furthermore, a large number of fat cells replaced the hematopoietic cells, and megakaryocytes were almost absent. In the transplant group, the myeloid, erythroid, and megakaryocytic cells were more visible and fat cells were reduced relative to the model group (Figure 4).

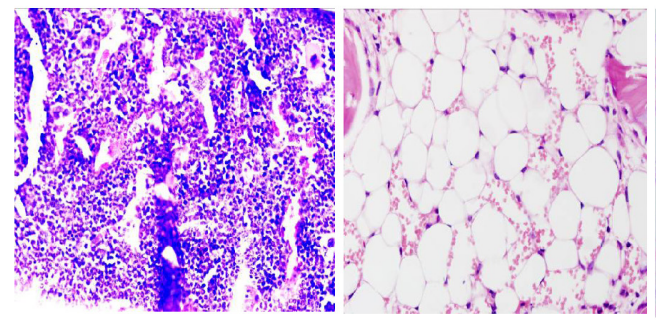

A
$\mathrm{B}$

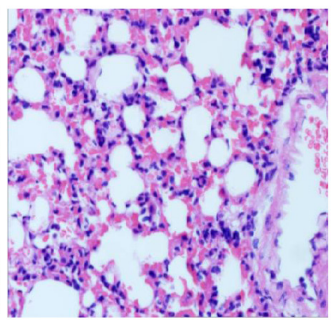

C

Figure 4. Pathological section of bone marrow in mouse femur (Wrigth-Giemsa's staining, 400X). A. Normal control mice; B. bone marrow failure mice; C. transplantation mice. 


\section{Dynamic distribution of CM-Dil-labeled BMSCs in bone marrow by flow cytometry}

At $24 \mathrm{~h}$ after BMSC transplantation, the ratio of CM-Dil positively labeled cells in the bone marrow was $0.26 \pm 0.02 \%$. At $72 \mathrm{~h}$ after BMSC transplantation, the ratio of CM-Dil positively-labeled cells in the bone marrow was $0.42 \pm 0.03 \%$, which was significantly higher than that of the 24-h group $(\mathrm{P}=0.01)$ (Figure 5).
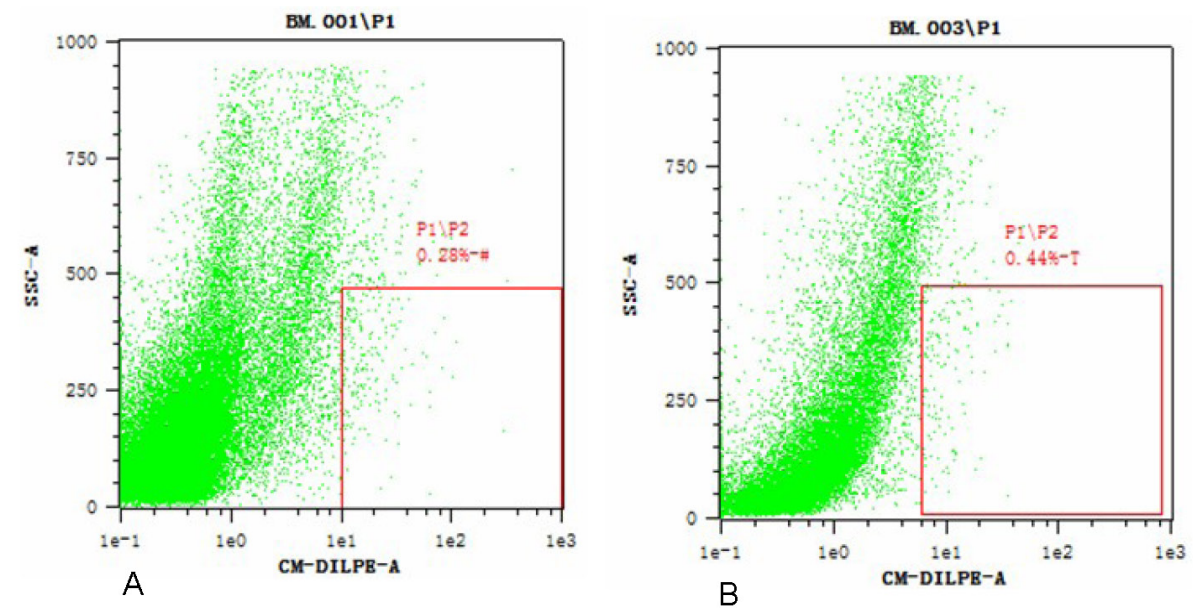

Figure 5. Flow cytometric analysis of chloromethyl-benzamidodialkylcarbocyanine-labeled cell proportion in bone marrow of transplantation mice. A. At $24 \mathrm{~h}$ after transplantation; B. at $72 \mathrm{~h}$ after transplatation.

\section{Homing and distribution of BMSCs in vivo under the fluorescence microscope}

Twenty-four hours after CM-Dil-labeled BMSC transplantation, a small amount of red CM-Dil fluorescently labeled cells was seen in bone marrow mononuclear cells, suggesting that BMSCs were homing to the bone marrow. At $72 \mathrm{~h}$ after transplantation, the red fluorescence of the bone marrow increased, but the fluorescence intensity remained unchanged, indicating that the mononucelar cells could migrate to injured bone marrow tissues.

\section{DISCUSSION}

AA is associated with hematopoietic stem cell proliferation or differentiation defects, an abnormal hematopoietic microenvironment, and immune dysfunction (Brodsky and Jones, 2005). Furthermore, the number of BMSCs of AA patients is reduced relative to those of normal humans. The hematopoietic supporting role has also been shown to be significantly reduced compared with normal BMSCs, its proliferative capacity was defected, and inhibition of activated T cells was lacking. BMSCs can adhere and secrete a variety of hematopoietic growth factors by inducing hematopoietic stem cells to play the hematopoietic supporting role (Jiang et al., 2005; Klyushnenkova et al., 2005). BMSCs also have immunoregulatory activity, which can reversibly inhibit the proliferation of T cells in mixed lymphocyte reactions. BMSCs inherently have low immunogenicity portability, which could be exploited for transplantations and play an important role in AA cell therapy (Ball et al., 2007; Weber-Mzell et al., 2007). 
Most researchers have adopted irradiation and lymphocyte infusion methods to establish AA animal models; however, the specific steps employed in these procedures are not standardized. The procedures described in the present study combine several approaches from the literature (Chen et al., 2004, 2005; Omokaro et al., 2009). After several preliminary experiments, we first exposed the BALB/c mice to $5.0 \mathrm{~Gy} \gamma$-ray irradiation and then injected $5 \times 10^{6}$ lymph node cells of DBA/2 mice to establish the animal model of bone marrow failure. This model had characteristics of high success rate and good stability.

The living cell tracer, CM-Dil, is a novel lipophilic carbocyanine dye with membrane permeability, which can remain in the cytoplasm during cell division, and emits red fluorescence at an excitation wavelength of $570 \mathrm{~nm}$. Compared with similar dyes, Dil and water-soluble PKH26, CM-Dil has good solubility in water, is a clear and simple mark with an intracellular residence time of up to 2 months, is resistant to embedded and fixed factors, and is suitable for long-term cell tracer studies (Hemmrich et al., 2006).

Previous experiments have simply spliced solid frozen tissues into slices to qualitatively observe the fluorescence distribution under a fluorescence microscope. In contrast, our experiment used flow cytometry with a fluorescence microscope to decompose the bone marrow into a single cell suspension sample, which could be added to tubes, thus reflecting the distribution of all positive cells in the bone marrow. Compared with the method of preparing and observing a large number of tissue slices, the method described herein is less labor intensive, detection is fast, efficiency is high, and it enables quantitative comparisons with sensitive and accurate identification.

Several studies have shown that when tissues are damaged, BMSCs from bone marrow or from the exogenous infusion are generally the first to localize to the inflammation area and damaged tissues with limited efficiency (Park et al., 2011; Cruz et al., 2012). In the model of bone marrow failure, ray irradiation can severely damage the bone marrow hematopoietic cells and the hematopoietic microenvironment. Therefore, the infusion of exogenous lymphocytes can cause even more severe bone marrow damage.

The experimental results showed that the BMSC infusion could localize to the bone marrow within $24 \mathrm{~h}$ in the bone marrow failure model. The BMSC content increased at $72 \mathrm{~h}$ in the bone marrow, suggesting that BMSCs were homing to the damaged bone marrow after infusion via blood circulation, and performed the repair function of the bone marrow microenvironment. Our experiment revealed that in the bone marrow failure model, untreated mice all died due to a decrease in blood cells and bone marrow failure. Most of the mice that survived after infusion of BMSCs showed restoration of blood cells and bone marrows, and improvement in overall condition.

Collectively, the results of the present study indicated that BMSCs may perform the roles of hematopoietic support, tissue repair, and local immune regulation (Huang et al., 2011) by homing to the bone marrow through direct contact between cells or indirectly, through the secretion of hematopoietic growth factors. These processes will ultimately promote bone marrow recovery, reduce the degree of bone marrow failure, significantly prolong survival time, and finally, show beneficial effects in bone marrow failure mouse models.

\section{ACKNOWLEDGMENTS}

Research supported by the National Natural Science Foundation of China (\#81101471/ 
H1602) and the PLA Medical Science and Technique Foundation during the 12th Five-Year Plan Period (\#BWS11J071).

\section{REFERENCES}

Ball LM, Bernardo ME, Roelofs H, Lankester A, et al. (2007). Cotransplantation of ex vivo expanded mesenchymal stem cells accelerates lymphocyte recovery and may reduce the risk of graft failure in haploidentical hematopoietic stemcell transplantation. Blood 110: 2764-2767.

Brodsky RA and Jones RJ (2005). Aplastic anaemia. Lancet 365: 1647-1656.

Chen J, Lipovsky K, Ellison FM, Calado RT, et al. (2004). Bystander destruction of hematopoietic progenitor and stem cells in a mouse model of infusion-induced bone marrow failure. Blood 104: 1671-1678.

Chen J, Brandt JS, Ellison FM, Calado RT, et al. (2005). Defective stromal cell function in a mouse model of infusioninduced bone marrow failure. Exp. Hematol. 33: 901-908.

Cruz M, Dissaranan C, Cotleur A, Kiedrowski M, et al. (2012). Pelvic organ distribution of mesenchymal stem cells injected intravenously after simulated childbirth injury in female rats. Obstet. Gynecol. Int. 2012: 612946.

Fang B, Li N, Song Y, Li J, et al. (2009). Cotransplantation of haploidentical mesenchymal stem cells to enhance engraftment of hematopoietic stem cells and to reduce the risk of graft failure in two children with severe aplastic anemia. Pediatr. Transplant. 13: 499-502.

Gao Y, Xiao Y and Jiang ZJ (2010). Efficacy and safety of bone marrow mesenchymal stem cell in the treatment of aplastic anemia. Guangdong Yixue 21: 2797-2800.

Hemmrich K, Meersch M, von HD and Pallua N (2006). Applicability of the dyes CFSE, CM-DiI and PKH26 for tracking of human preadipocytes to evaluate adipose tissue engineering. Cells Tissues Organs 184: 117-127.

Huang Y, Zheng CL and Yang SG (2011). Mesenchymal stem cells in the bone marrow of immune-mediated aplastic anemia treatment. Zhongguo Zuzhi Gongcheng yu Linchuang Kangfu 15: 41-45.

Jiang XX, Zhang Y, Liu B, Zhang SX, et al. (2005). Human mesenchymal stem cells inhibit differentiation and function of monocyte-derived dendritic cells. Blood 105: 4120-4126.

Klyushnenkova E, Mosca JD, Zernetkina V, Majumdar MK, et al. (2005). T cell responses to allogeneic human mesenchymal stem cells: immunogenicity, tolerance, and suppression. J. Biomed. Sci. 12: 47-57.

Kruyt MC, De BJ, Veenhof M, Oner FC, et al. (2003). Application and limitations of chloromethylbenzamidodialkylcarbocyanine for tracing cells used in bone Tissue engineering. Tissue Eng. 9: 105-115.

Ma HY, Xiao R and Du XY (2011). Fluorescent reactive dye (CM-Dil) human bone marrow mesenchymal stem cell proliferation. Kouqiang Hemian Waike Zazhi 4: 251-255.

Omokaro SO, Desierto MJ, Eckhaus MA, Ellison FM, et al. (2009). Lymphocytes with aberrant expression of Fas or Fas ligand attenuate immune bone marrow failure in a mouse model. J. Immunol. 182: 3414-3422.

Park BN, Shim W, Lee G, Bang OY, et al. (2011). Early distribution of intravenously injected mesenchymal stem cells in rats with acute brain trauma evaluated by (99m)Tc-HMPAO labeling. Nucl. Med. Biol. 38: 1175-1182.

Tang Y, Desierto MJ, Chen J and Young NS (2010). The role of the Th1 transcription factor T-bet in a mouse model of immune-mediated bone-marrow failure. Blood 115: 541-548.

Weber-Mzell D, Urban C, Benesch M, Rojacher T, et al. (2007). Durable remission following a third allogeneic stem cell transplantation in a patient with repeatedly relapsing SAA. The importance of stroma cells for sustained engraftment? Pediatr. Transplant. 11: 332-335.

Xiao Y, Jiang ZJ, Pang Y, Li L, et al. (2013). Efficacy and safety of mesenchymal stromal cell treatment from related donors for patients with refractory aplastic anemia. Cytotherapy 15: 760-766.

Yao J and Li SN (1991). Lymphocytes and experimental study of the relationship of aplastic anemia. Zhonghua Xueyexue Zazhi 12: 229-231. 